\title{
Impact of ethyl pyruvate on Adriamycin-induced cardiomyopathy in rats
}

\author{
MENGLIN LIU ${ }^{1 *}$, MENGLONG WANG ${ }^{2 *}$, JIANFANG LIU $^{2}$, ZHEN LUO $^{2}$,

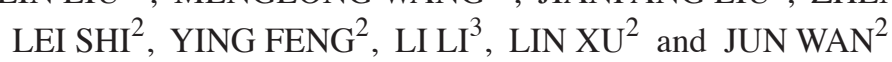 \\ Departments of ${ }^{1}$ Emergency and ${ }^{2}$ Cardiology, Renmin Hospital of Wuhan University, Wuhan, Hubei 430060, P.R. China; \\ ${ }^{3}$ Department of Psychiatry and Behavioral Neurobiology, University of Alabama at Birmingham, \\ Birmingham, AL 35294, USA
}

Received May 26, 2016; Accepted September 9, 2016

DOI: $10.3892 /$ etm.2016.3795

\begin{abstract}
Ethyl pyruvate (EP), a derivative of pyruvic acid, is known to have protective effects against ischemic cardiomyopathy and other disorders. However, little is known about its role in Adriamycin (ADR)-induced cardiomyopathy. The present study was designed to investigate the impact of EP on ADR-induced cardiomyopathy in an animal model. Sixty male Sprague-Dawley (SD) rats were divided into four groups: Normal control, EP, ADR and ADR + EP groups (n=15/group). Rats in the ADR and ADR + EP groups were treated with $\operatorname{ADR}(2.5 \mathrm{mg} / \mathrm{kg} /$ week intraperitoneally) for 6 weeks. From the eighth week, rats in the EP and ADR + EP groups received EP via gastric lavage at a dose of $50 \mathrm{mg} / \mathrm{kg} /$ day for 30 days. After completing the EP treatment, cardiac function was assessed by echocardiography and then rats were sacrificed. Hearts were harvested for subsequent analysis. Compared with rats in the normal control and EP groups (without ADR treatment), rats in the ADR and ADR + EP groups showed significant impairments in terms of cardiac function, apoptosis, severe oxidative stress and fibrosis in the heart. However, these impairments were alleviated by EP treatment in the ADR + EP group. Upon EP treatment, cardiac function was significantly improved. The levels of oxidative stress, fibrosis and apoptosis in the myocardial tissues were also significantly reduced. These findings indicated that EP treatment attenuated, at least partially, ADR-induced cardiomyopathy in rats.
\end{abstract}

Correspondence to: Dr Jun Wan, Department of Cardiology, Renmin Hospital of Wuhan University, 238 Jiefang Road, Wuhan, Hubei 430060, P.R. China

E-mail: wanjun1963@126.com

*Contributed equally

Key words: ethyl pyruvate, Adriamycin, oxidative stress, fibrosis, apoptosis

\section{Introduction}

Adriamycin (ADR), an anthracycline, is an effective chemotherapeutic agent. However, ADR is cardiotoxic and may cause dose-dependent, progressive cardiac damage which is clinically manifested as reduced left ventricular ejection fraction and ultimately heart failure (1). Moreover, chronic ADR-induced cardiomyopathy is associated with a poor prognosis and low survival rate (1). One of the options to prevent cardiotoxicity is a dose-limiting approach or limiting the use of ADR, however, that may also compromise its chemotherapeutic properties. In recent years, studies have been conducted to elucidate the mechanisms underlying ADR-induced cardiomyopathy and to explore interventional strategies, and several mechanisms have been hypothesized to account for the ADR-induced cardiotoxicity, including oxidative stress, imbalance of $\mathrm{Ca}^{2+}$ in the cytoplasm and mitochondria, apoptosis and fibrosis $(2,3)$. However, a definite mechanism remains unclear.

Pyruvate, the anionic form of a simple $\alpha$-keto acid, is normally regarded as a key intermediate in the oxidative or anaerobic metabolism of glucose. Pyruvate has been shown to ameliorate organ damage, such as ischemic cardiomyopathy as well as ischemic-reperfusion injury of heart (4), intestine (5), liver (6), and brain (7), although the underlying mechanisms are not well defined. In addition, pyruvate also plays an important role in combatting oxidative damage by scavenging hydrogen peroxide and lipid peroxides and by increasing the antioxidant redox potential of the endogenous glutathione system (8). Furthermore, pyruvate has also been shown to resist apoptosis and fibrosis in various tissues $(9,10)$. However, due to aqueous instability, the use of pyruvate as a therapeutic agent is limited. Ethyl pyruvate (EP), an ester form of pyruvic acid (11), is used as a practical pyruvate precursor for administration and has better protective effects on some damaged organs because of its stable chemical structure (5).

Therefore, EP may be able be to act as a metabolic substrate and an effective reactive oxygen species (ROS) scavenger. In the present study, it was hypothesized that EP has protective effects against ADR-induced cardiomyopathy in an animal model. Experiments were performed to examine the protective effects of EP in a rat model, in which chronic myocardial injury was induced by ADR. In this particular rat model, cardiac 
geometry and function, myocardial tissue oxidative stress and fibrosis, and myocardial cell apoptosis were assessed.

\section{Materials and methods}

Animals. All procedures involving animals were approved by the Ethics Committee for Animal Research of Wuhan University (Wuhan, China). All animals received humane care in compliance with the Guide for the Care and Use of Laboratory Animals prepared by the Institute of Laboratory Animal Resources and the National Research Council (12). Male Sprague-Dawley (SD) rats, aged 6-8 weeks and weighing 150-180 g, were purchased from the Experimental Animal Center of Wuhan University. All animals were acclimated to the laboratory for 7 days prior to the experiment and were maintained in a light-controlled room (12-h light/dark cycle) at an ambient temperature of $25^{\circ}$ with free access to water and standard chow.

Sixty male SD rats were randomly divided into four groups, namely the control group, ethyl pyruvate (EP) group, Adriamycin (ADR) group and ADR + EP group ( $n=15$ per group). Rats in the ADR and ADR + EP groups were treated with ADR (Actavis Italy SpA, Nerviano, Italy) at a dose of $2.5 \mathrm{mg} / \mathrm{kg} /$ week via intraperitoneal injection for 6 weeks. By contrast, rats in the control and EP groups were treated with normal saline (via intraperitoneal injection) at the same dose as ADR for 6 weeks. From the eighth week, rats in the EP group and the ADR + EP group received EP (Sigma-Aldrich; Merck Millipore, Darmstadt, Germany) via stomach lavaging at a dose of $50 \mathrm{mg} / \mathrm{kg} / \mathrm{day}$ for 30 days. Upon the completion of 30 days treatment with EP, cardiac function was accessed by echocardiography. Rats were then sacrificed by an overdose of anesthesia $(3.5 \mathrm{mg} / 100 \mathrm{~g}$ pentobarbital) for the harvesting of heart tissues.

Echocardiography test. Echocardiography was performed using a high-resolution ultrasound imaging system equipped with a 7V3 probe with a frequency of $6.0 \mathrm{MHz}$ (Acuson Sequoia; Siemens, Washington, DC, USA). Fractional shortening (FS), left ventricular internal dimension diastolic (LVIDD) and left ventricular internal dimension systole (LVIDS) were recorded from the parasternal long-axis M-mode images using averaged measurements from 3-5 consecutive cardiac cycles. End diastolic volume (EDV) and end systolic volume (ESV) were calculated from bi-dimensional long-axis parasternal views by means of the single-plane area-length method. The ejection fraction $(\mathrm{EF})$ was calculated as follows: $\mathrm{EF}(\%)=(\mathrm{LVEDV}-\mathrm{L}$ VESV) / LVEDV x 100.

Histological examination and determination of apoptosis. Myocardial tissues excised by horizontal intercept from the middle part of the whole heart were fixed in $10 \%$ buffered formalin for $24 \mathrm{~h}$, and then embedded in paraffin and sliced into $5-\mu \mathrm{m}$ sections. The sections were stained with picrosirius red (PSR) to identify collagen deposition, and then were visualized using light microscopy. Fibrillar collagen was visualized under the microscope and the left ventricular collagen volume fraction was measured using a quantitative digital image analysis system (Image-Pro Plus 6.0; Media Cybernetics, Inc., Rockville, MD, USA). The cardiomyocyte apoptosis rate was also assessed using a terminal deoxynucleotidyl transferase-mediated dUTP nick end-labeling (TUNEL) assay.

Briefly, sections (3- $\mu \mathrm{m}$-thick) from formalin-fixed and paraffin-embedded myocardial tissue were deparaffinized with xylene and dehydrated with ethanol. Slides were rinsed twice with PBS and treated with proteinase $\mathrm{K}(15 \mathrm{~g} / \mathrm{ml}$ in $10 \mathrm{mM}$ Tris/HCl; pH 7.4-8.0) for $15 \mathrm{~min}$ at $37^{\circ}$. Endogenous peroxidases were blocked with $3 \%$ hydrogen peroxide in methanol at room temperature for $10 \mathrm{~min}$. Tissue sections were analyzed with an in situ cell death detection kit (Roche Diagnostics, Indianapolis, IN, USA), in accordance with the manufacturer's instructions. Reactions were visualized with fluorescence microscopy and were measured using a quantitative Image-Pro Plus 6.0 digital image analysis system.

Reverse transcription-quantitative polymerase chain reaction (RT-qPCR) and western blotting. RT-qPCR and western blotting were performed as previously described (13). Briefly, after total RNA was extracted from ventricles using TRIzol reagent (Invitrogen; Thermo Fisher Scientific, Inc., Waltham, MA, USA), first strand cDNA was synthesized using a Transcriptor First Strand cDNA Synthesis kit (Roche Diagnostics). qPCR was performed using SYBR Green PCR Master Mix (Roche Diagnostics) to determine the expression levels of genes of interest which were transforming growth factor- $\beta 1$ (TGF $\beta-1)$, collagen type $1 \alpha 1$ (Colla1), collagen type $1 \alpha 3$ (Colla3), tissue inhibitor of metalloproteinase (TIMP)1, TIMP2, matrix metalloproteinase (MMP)2 and MMP9, and the results were normalized against GAPDH gene expression. PCR cycling conditions were as follows: Predenaturation at $95^{\circ} \mathrm{C}$ for $10 \mathrm{~min}$, followed by 40 cycles at $95^{\circ} \mathrm{C}$ for $15 \mathrm{sec}, 60^{\circ} \mathrm{C}$ for $1 \mathrm{~min}$ and $72^{\circ} \mathrm{C}$ for $20 \mathrm{sec}$, and a final extension at $60^{\circ} \mathrm{C}$ for $5 \mathrm{~min}$. The PCR primers that were used are shown in Table I. PCR reactions were repeated twice.

For western blotting, cardiac tissue was lysed in radioimmunoprecipitation assay buffer (Roche Diagnostics). Protein extracts (30 $\mu \mathrm{g}$ per lane) were separated by $10 \%$ SDS-PAGE, transferred to polyvinylidene difluoride (PVDF) membranes and probed with primary antibodies overnight at $4^{\circ} \mathrm{C}$. The primary antibodies included anti-GAPDH (1:1,000; sc-365062), anti-NADPH oxidase-4 (Nox4; 1:1,000; sc-517188), anti-Bax (1:1,000; sc-23959); anti-Bcl-2 (1:1,000; sc-7382) and anti-caspase-3 (1:1,000; sc-65496; Santa Cruz Biotechnology, Inc., Dallas, TX, USA) and anti-NADPH oxidase-2 (Nox2; (1:1,000; ab80508; Abcam, Cambridge, UK). Following washing with TBST three times, the membranes were incubated with horseradish peroxidase-conjugated goat anti-rabbit/mouse secondary antibodies (1:100; G1201; Guge Biotechnology Co., Ltd., Wuhan, China) for $1 \mathrm{~h}$ at room temperature. Subsequently, the membranes were treated with ECL reagents (Bio-Rad Laboratories, Inc., Hercules, CA, USA) prior to visualization using a FluorChem E imager (ProteinSimple, San Jose, CA, USA) according to the manufacturer's instructions. The specific protein expression levels were normalized to the levels of GAPDH on the same PVDF membrane.

Evaluation of superoxide dismutase (SOD) activity and malondialdehyde (MDA) concentration. The activity of SOD in 
Table I. Primers for quantitative polymerase chain reaction.

\begin{tabular}{lll}
\hline Primer name & \multicolumn{1}{c}{ Forward primer } & \multicolumn{1}{c}{ Reverse primer } \\
\hline GAPDH-rat & ACGGGAAACCCATCACCATC & TGGTGGTGCAGGATGCATTG \\
TGF 31 -rat & GCGCCTGCAGAGATTCAAGTCAAC & GTATCAGTGGGGGTCAGCAGCC \\
COL1a1-rat & TGGCAACCTCAAGAAGTCCC & ACAAGCGTGCTGTAGGTGAA \\
COL1a3-rat & CAACCAGTGCAAGTGACCAA & GCACCATTGAGACATTTTGAAG \\
MMP2-rat & CTGATAACCTGGATGCCGTCGT & TGCTTCCAAACTTCACGCTCTT \\
MMP9-rat & TTATTGTGAGCATCCCTAGGG & AGTGTCCGAGGAAGATACTTG \\
TIMP-1-rat & ACAGCTTTCTGCAACTCGGA & CCGGAAACCTGTGGCATTTC \\
TIMP-2-rat & CTAATTGCAGGGAAGGCGGA & CATAGGGCAGCGTGTGATCT
\end{tabular}

TGF- $\beta 1$, transforming growth factor- $\beta 1$; COL1a1, collagen type $1 \alpha 1$; COL1a3, collagen type $1 \alpha 3$; MMP, matrix metalloproteinase; TIMP, tissue inhibitor of metalloproteinase.

myocardial tissue was detected by the xanthine oxidase technique. This procedure is based upon the inhibition of nitrite (NIT) reduction due to the superoxide anion generated by the combination of xanthine and xanthine oxidase. An SOD assay kit (Nanjing Jiancheng Bioengineering Institute, Nanjing, China) was used to assess the SOD activity. One unit of SOD was the amount that caused a 50\% inhibition in the rate of NIT reduction. The SOD activity was expressed as $\mathrm{U} / \mathrm{mg}$ protein in myocardial tissue homogenate.

The content of MDA in myocardial tissue was assayed according to thiobarbituric acid (TBA) method. In this method MDA reacts with TBA under high temperature $\left(90-100^{\circ} \mathrm{C}\right)$ and acidic conditions to form TBA reactive substances (TBARS). TBARS were measured using a spectrophotometer at $532 \mathrm{~nm}$. An MDA assay kit (Nanjing Jiancheng Bioengineering Institute) was used to assess the MDA concentration. The content of MDA was expressed in units of $\mathrm{nmol} / \mathrm{mg}$ protein in myocardial tissue homogenate.

Statistical analysis. All statistical analyses were performed using SPSS software, version 18.0 (SPSS, Inc., Chicago, IL, USA). The inter-group differences were analyzed by one-way analysis of variance. The data are expressed as the mean + standard deviation. All P-values were two-sided and $\mathrm{P}<0.05$ was considered to indicate a statistically significant difference.

\section{Results}

Mortality of rats. Out of 60 rats, 51 completed the study. The mortality rates of the ADR group and the ADR + EP group were 33.3 and $26.7 \%$, respectively, at the end of the interventions, while no deaths were encountered in other groups.

Cardiac functions. Echocardiography was performed in each rat to measure relative parameters of cardiac functions. As shown in Fig. 1A, two-dimensional and M-mode short-axis views of the left ventricle were acquired at the level of the papillary muscles in rats. There was no difference in terms of LVIDD and EVD between the four groups (data not shown). Compared with the control group, treatment with EP did not affect FS, EF, LVIDS and ESV (Fig. 1B-E). By contrast, FS and $\mathrm{EF}$ in the ADR + EP group were significantly higher than those in the ADR group (Fig. 1B and C). Moreover, LVIDS and ESV in the ADR + EP group were greatly lower than those in the ADR group (Fig. 1D and E), indicating that treatment with EP improved the impaired cardiac functions induced by ADR in rats.

Differential effects of ADR and EP on oxidative stress-related molecules. SOD is the major defense against ROS production in cells (14), and MDA is produced by the actions of ROS on the lipids existing in the membranes of the cells (15). Therefore, SOD and MDA can be used to experimentally evaluate oxidative injury. To understand the mechanisms underlying the protective role of EP against ADR-induced cardiotoxicity, MDA levels and SOD activity of myocardial tissues were measured in rats. Treatment with EP alone did not affect the production of MDA and SOD compared with that in the control group (Fig. 2A and B). However, in the ADR group, the SOD activity of the myocardial tissue was significantly lower (Fig. 2A), while the MDA level was significantly higher than those in control group, EP group and ADR + EP group (Fig. 2B).

Another two molecules associated with oxidative stress, namely Nox 2 and Nox4, were also investigated in rats using western blot analysis. The protein level of Nox4 in the cardiomyocytes in the ADR group was significantly higher than those in the control group, EP group and ADR + EP group, and was reduced by EP (Fig. $2 \mathrm{C}$ and D). By contrast, there was no significant difference in the protein levels of Nox 2 among the four groups (Fig. 2C and E).

EP reduces ADR-induced myocardial cell apoptosis. To assess the effects of ADR and EP on myocardial cell apoptosis, markers of apoptosis were assessed in this study. TUNEL staining showed an increase in cardiomyocyte apoptosis rate in the ADR group, whereas the increased apoptosis rate was reduced by EP treatment in the ADR + EP group (Fig. 3). No apoptosis was observed in the control and EP only groups (Fig. 3). To confirm these findings, western blot analysis was applied to assess the expression levels of the apoptosis-related proteins caspase-3, Bax and Bcl-2. As shown in Fig. 4, the expression levels of casepase-3 and Bax 
A

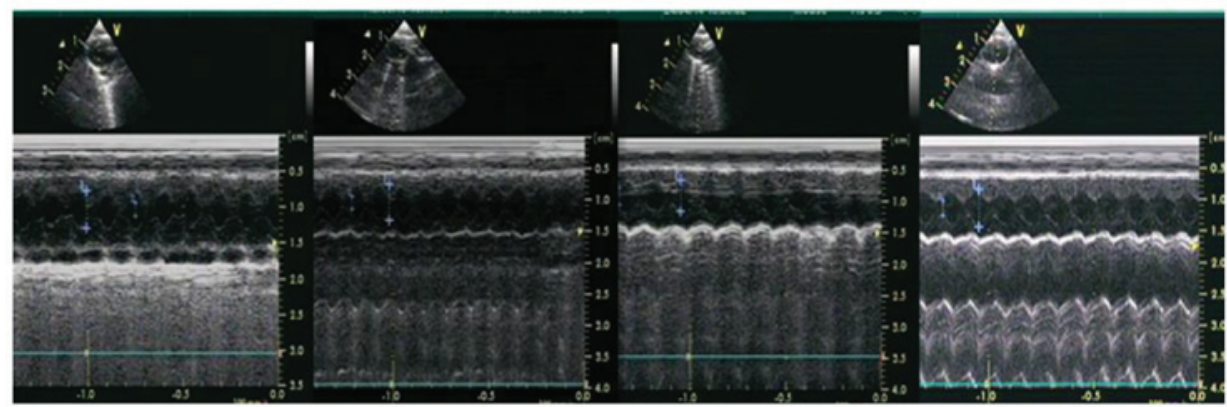

B

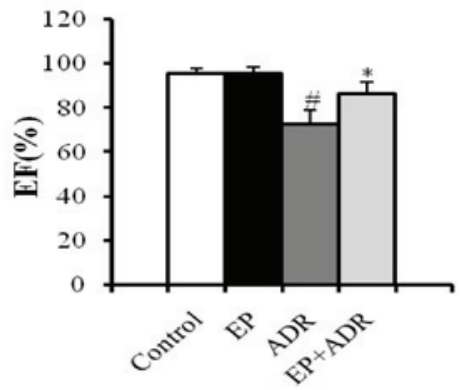

D

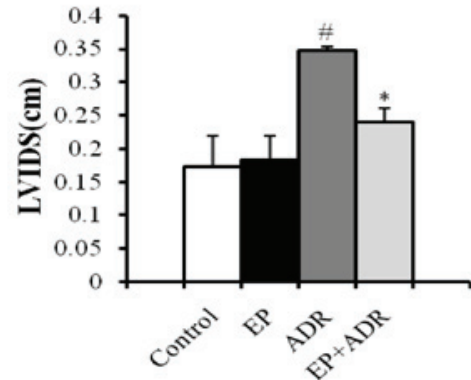

C

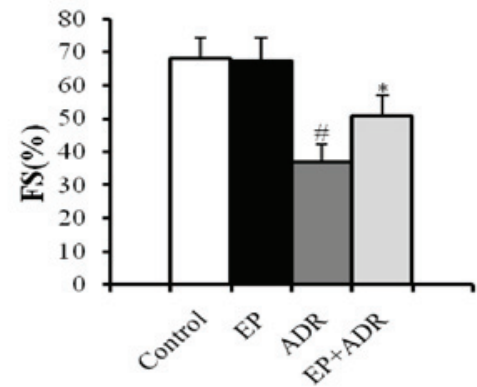

$\mathbf{E}$

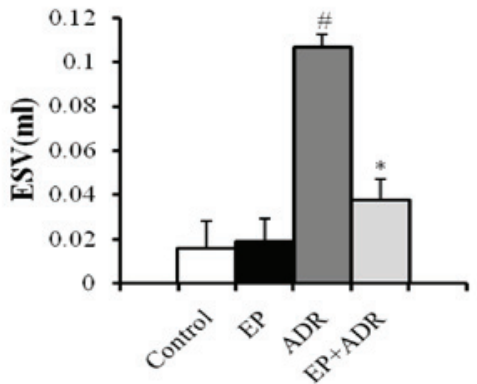

Figure 1. Results of echocardiography indicate that EP can improve the heart functions of rats damaged by ADR. (A) Two-dimensional (upper panel) and M-mode (lower panel) short-axis views of the left ventricle at the level of the papillary muscles in four different animals from the control (first), EP (second), ADR (third) and EP + ADR (last) groups, respectively. Results of (B) EF, (C) FS, (D) LVIDS and (E) ESV in the four groups. "P<0.05 vs. control and EP groups, ${ }^{*} \mathrm{P}<0.05$ vs. ADR group. EP, ethyl pyruvate; ADR, Adriamycin; EF, ejection fraction. FS, fractional shortening; LVIDS, left ventricular internal dimension systole; ESV, end systolic volume.

in myocardial tissue were significantly higher in the ADR group than in the control group, EP group and ADR + EP group. By contrast, the expression level of Bcl-2 was significantly lower in the ADR group than in the other three groups (Fig. 4C).

EP attenuates ADR-induced myocardial tissue fibrosis. The consequence for ADR-induced chronic damage is myocardial tissue fibrosis. Thus, to explore whether treatment with EP can attenuate ADR-induced myocardial tissue fibrosis, PSR staining and RT-qPCR assays were applied to evaluate the fibrosis in myocardial tissues. PSR staining in left ventricular tissue sections showed that the collagen volume fraction (\%) in the ADR group was significantly higher than those in the control group, EP group and ADR + EP group (Fig. 5). Furthermore, the results of RT-qPCR indicated that the mRNA levels of TGF $\beta-1$, Colla1 and Colla3, TIMP1, TIMP2, MMP2 and MMP9 in myocardial tissue in the ADR group were significantly higher than those in the other three groups (Fig. 6A-E). The ratios MMP9/TIMP1 and MMP2/TIMP2 in the ADR group were notably lower than those in the ADR + EP group (Fig. 6F and G), however, they were not significantly different from those in the control group and the EP group (Fig. 6F and G).

\section{Discussion}

ADR is known to be cardiotoxic because it induces degenerative myocardial lesions and cardiac dysfunction. Therefore, ADR has been used to establish models of dilated cardiomyopathy (16) and acute (17) or chronic (18) heart failure in many studies. In the present study, lower FS\% and decreased EF\% were observed in the ADR group, while the LVIDS and ESV were distinctly larger than those in the control group. By contrast, treatment with EP reversed the abnormalities in the indices FS\%, EF\%, LVIDS and ESV. However, there were no significant differences of LVIDD and EDV among the four groups.

It has previously been suggested that the severity and timing of degenerative myocardial lesions and cardiac dysfunction affect the mortality of rats (19). In the present study, the highest mortality rate was observed in the ADR group, followed by the ADR + EP group, indicating that EP reduced the ADR-induced mortality. However, the reports of ADR-induced mortality by different groups are heterogeneous. A mortality rate of $14.3 \%$ was reported in one study, in which the same dose and frequency for ADR was used (16), while another study reported no mortality with the same total dose (20). These different results for mortality could be 

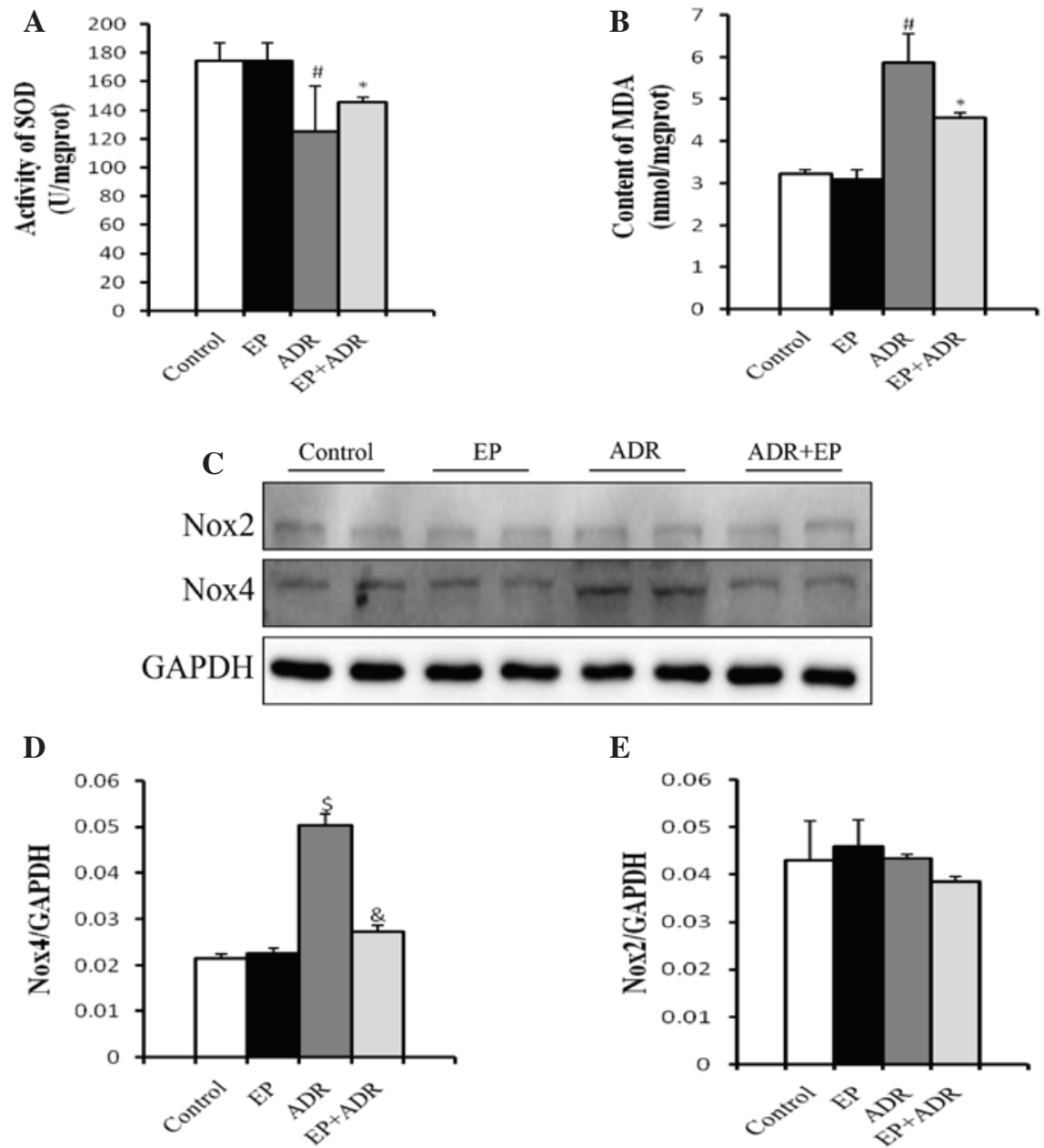

Figure 2. Differential effects of ADR and EP on oxidative stress-related molecules. (A) SOD activity and (B) MDA levels in myocardial tissue. (C) Western blot results for Nox4 and Nox 2 in each group, and quantified results for (D) Nox4 and (E) Nox2. "P<0.05 vs. control and EP groups, "P<0.05 vs. ADR group, ${ }^{\$} \mathrm{P}<0.01$ vs. control and EP groups, ${ }^{\&} \mathrm{P}<0.01$ vs. ADR group. EP, ethyl pyruvate; ADR, Adriamycin; SOD, superoxide dismutase; MDA, malondialdehyde; Nox2, NADPH oxidase-2; Nox4, NADPH oxidase-4.
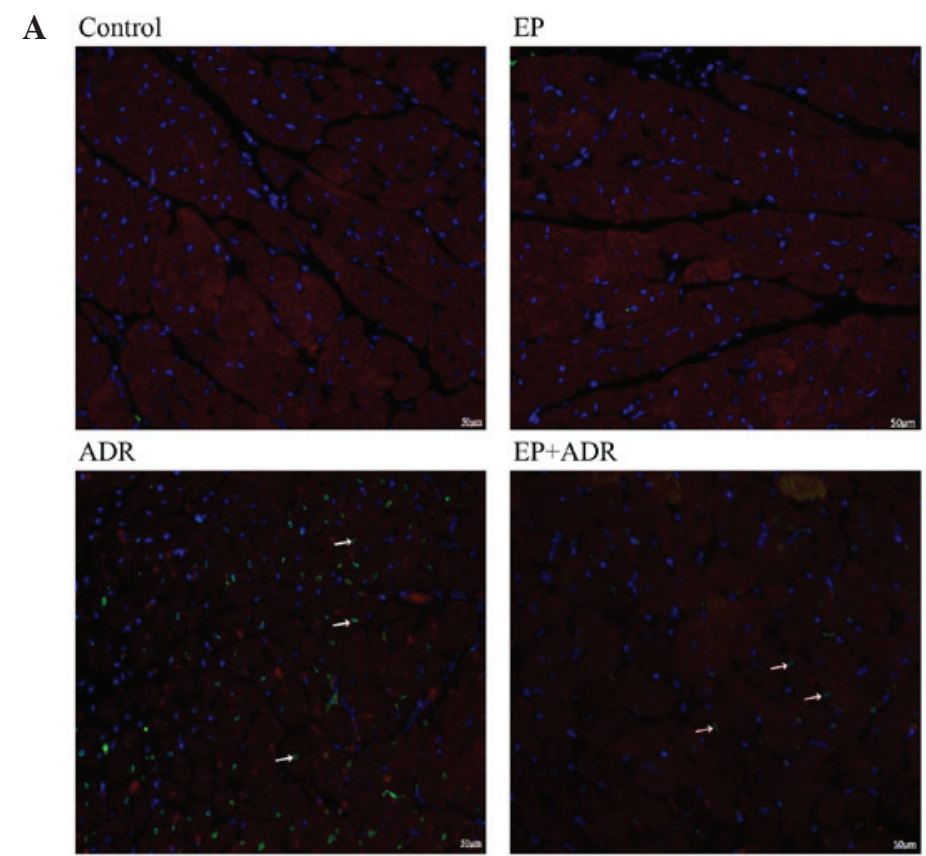

$\mathrm{EP}+\mathrm{ADR}$

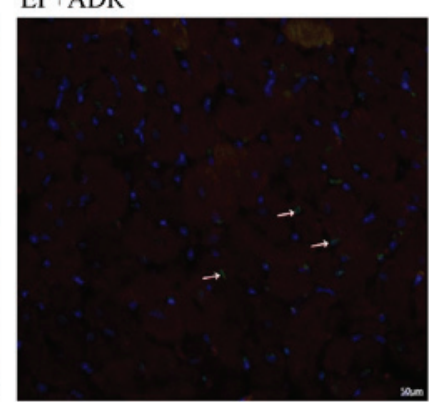

\section{B}

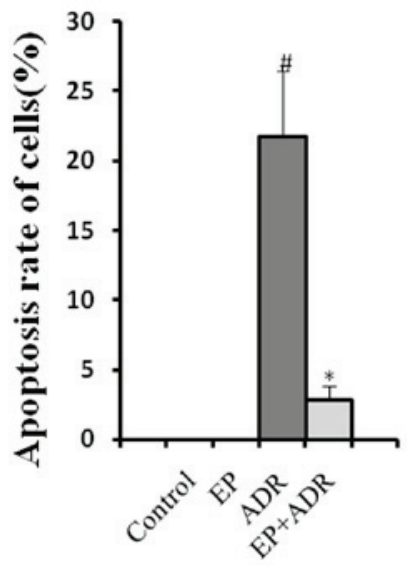

Figure 3. EP reduces ADR-induced myocardial cell apoptosis. (A) Images of TUNEL staining in which the arrows point to apoptotic myocardial cells. (B) Statistical results for cardiomyocyte apoptosis rate in the ADR and EP + ADR groups. ${ }^{\text {}} \mathrm{P}<0.05$ vs. control and EP groups; ${ }^{*} \mathrm{P}<0.05$ vs. ADR group. EP, ethyl pyruvate; ADR, Adriamycin; TUNEL, terminal deoxynucleotidyl transferase-mediated dUTP nick end labeling. 

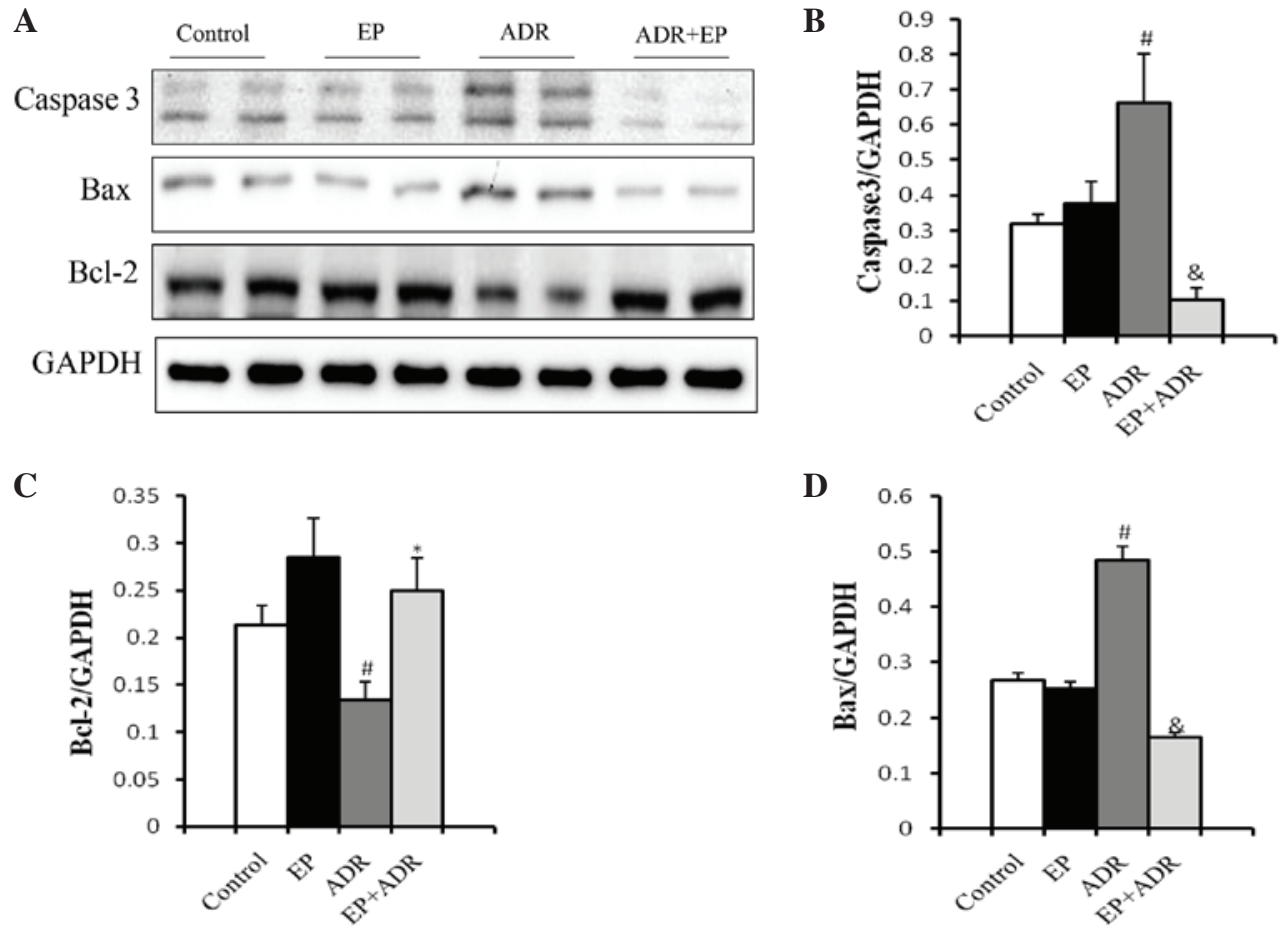

Figure 4. Effect of EP on expression levels of apoptosis-related proteins. (A) Western blots of caspase-3, Bcl-2 and Bax from myocardial tissue and the quantified expression levels of (B) caspase-3, (C) Bcl-2 and (D) Bax. " $\mathrm{P}<0.05$ vs. control and EP groups, " $\mathrm{P}<0.05$ vs. ADR group. ${ }^{\text {P }} \mathrm{P}<0.01$ vs. ADR group. EP, ethyl pyruvate; ADR, Adriamycin.

explained by the differences in dosage and protocols of ADR administration, experimental methods and animal conditions. Despite inconsistent reports, the data from the present study suggest that treatment with EP is able to alleviate the cardiotoxicity of ADR and thus improve the cardiac function and survival rate of rats.

Although ADR is known to be cardiotoxic, the mechanisms are not well established. Several theories are postulated and one of them is associated with the formation of ROS and myocardial oxidative injury. The NADPH oxidases are important sources of cellular ROS (21). In the Nox family, there are seven different homologs of Nox enzymes in mammalian genomes (22), named as Nox1 to Nox5 and Duox1 and Duox2. Moreover, Nox 2 and Nox4 are abundantly expressed in cardiomyocytes (23). Zhao et al (24) found that Nox2-deficient mice exhibited less oxidative injury of myocardial tissue in response to ADR. Moreover, Ortiz et al (25) reported that ADR induces ROS production by upregulating the expression of Nox4. Consistent with this, it was observed in the present study that ADR upregulates the protein expression of Nox4 in myocardial tissue, which was suppressed by EP. However, no effects of ADR or EP on the protein expression of Nox 2 were identified. Furthermore, it was observed that ADR reduced the activity of SOD and increased the levels of MDA in cardiomyocytes, while EP reversed these changes. Thus, these findings indicate that EP is able to inhibit ADR-induced oxidative injury, which might occur through downregulation of the expression of Nox4 and improvement of anti-oxidant activity.

Some studies have reported that ADR induces apoptosis in cardiomyocytes by upregulating the expression of Bax (a pro-apoptotic molecule) and caspase-3 and downregulating the expression of Bcl-2 (an anti-apoptotic molecule) $(26,27)$, which was further supported by the current study. In addition, the cardiomyocyte apoptosis rate in the EP + ADR group was reduced, which was accompanied by downregulated protein expression of Bax and caspase-3 and upregulated protein expression of Bcl-2. Thus, EP can protect against ADR-induced cardiotoxicity via changes in the expression of apoptosis-related proteins and reducing myocardial cell apoptosis.

Fibrosis is characterized as the excessive deposition of extracellular matrix components, which mainly consist of collagen (28), and can be regulated by many factors, including TGF $\beta-1$, MMPs and TIMPs. TGF $\beta$ - 1 can directly induce the expression of collagen proteins (29) and promote extracellular matrix deposition. MMPs are able to degrade extracellular matrix components, while TIMPs inhibit the degradation of extracellular matrix components (30). Therefore, upregulating the mRNA level of TIMPs or downregulating the mRNA levels of TGF $\beta-1$ and MMPs may inhibit fibrosis. Fibrosis is reportedly induced by ADR in myocardial tissue (31), which is supported by the present study findings. The present results indicate that EP can alleviate ADR-induced fibrosis by upregulating the mRNA levels of MMPs relative to those of TIMPs as well as downregulating the mRNA levels of TGF $\beta-1$ and TIMPs, since it was observed that rats in the ADR + EP group had a lower collagen volume fraction in myocardial tissue, lower mRNA levels of TGF $\beta$-1, TIMP1, TIMP2, Colla1 and Colla3, and higher ratios of MMP9/TIMP1 and MMP2/TIMP2, compared with those in the ADR group. However, the mRNA levels of MMP2 and MMP9 in the ADR group were higher than those in the other groups, which may due to the compensatory response of the myocardium to ADR treatment. 
A

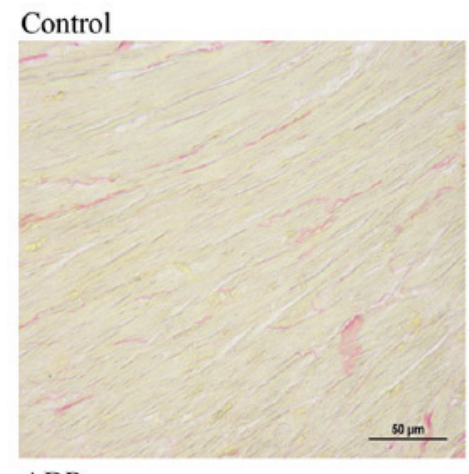

\section{ADR}

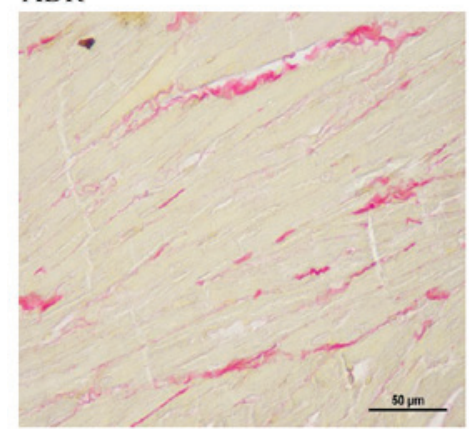

EP

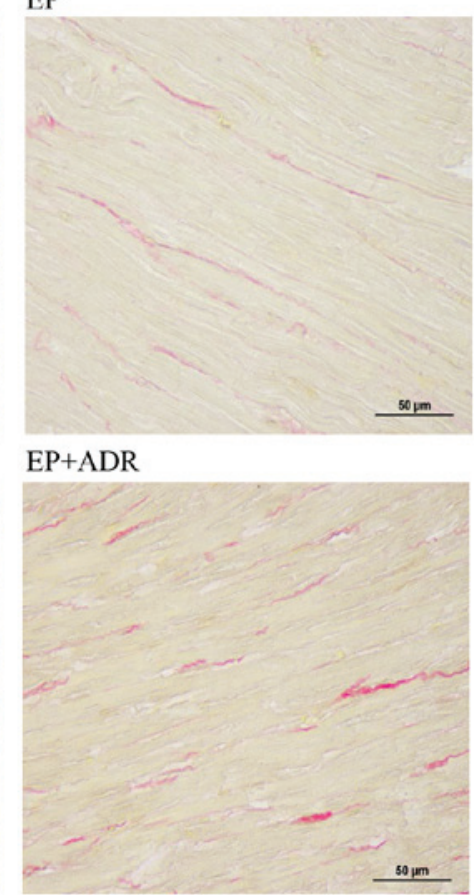

B

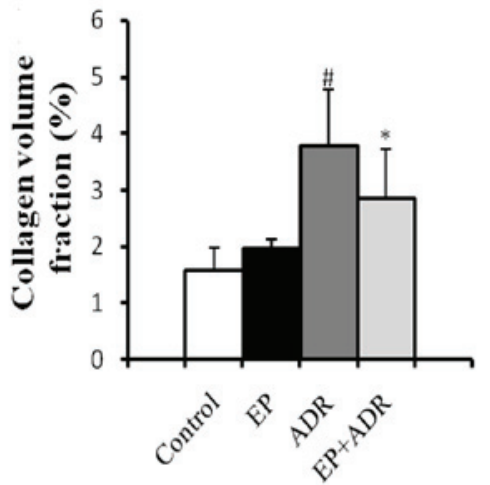

Figure 5. Histological analyses of picrosirius red staining. (A) Images of stained tissue and (B) quantified collagen volume fraction. ${ }^{\#} \mathrm{P}<0.05 \mathrm{vs}$. control and EP groups, "P<0.05 vs. ADR group. EP, ethyl pyruvate; ADR, Adriamycin.

A

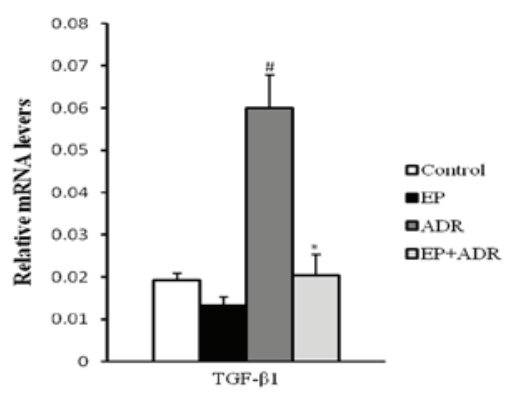

C

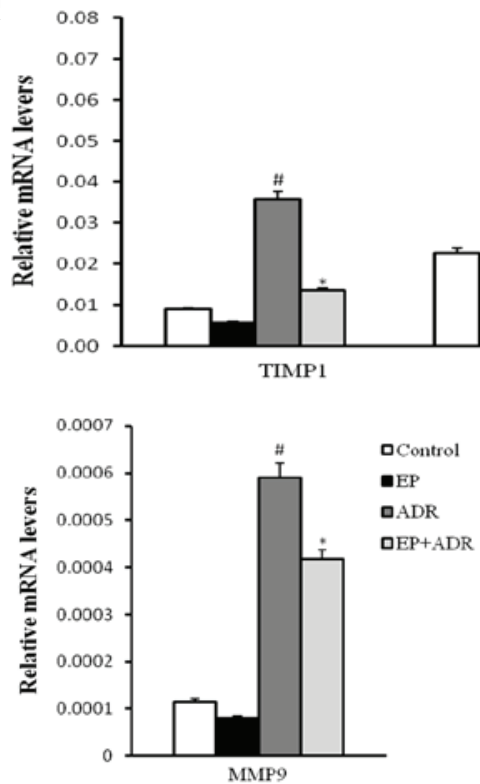

B

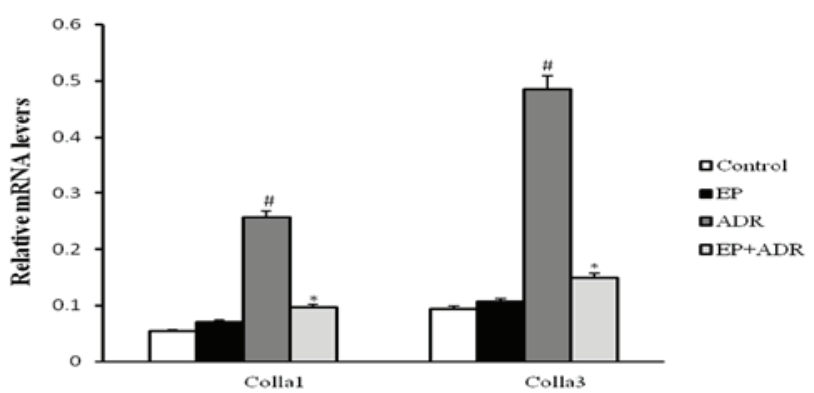

D

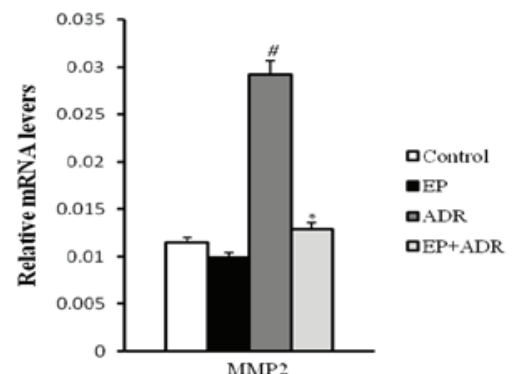

TIMP2

$\mathbf{F}$

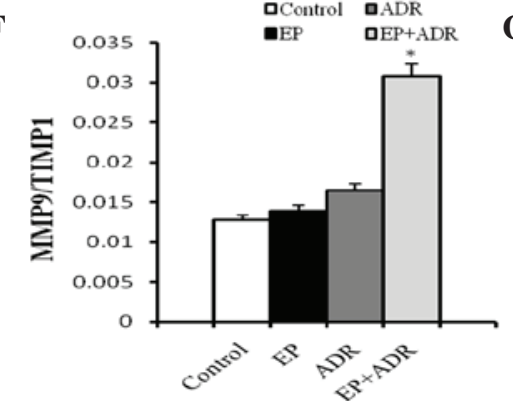

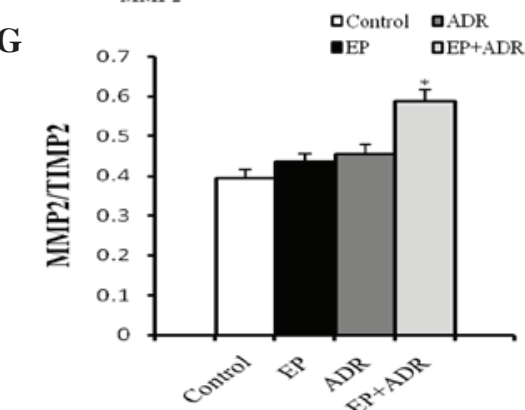

Figure 6. Results of RT-PCR analysis. The mRNA level of (A) TGF- $\beta 1$, (B) Colla1 and Colla3, (C) TIMP1 and TIMP2, (D) MMP2 and (E) MMP9, and the ratios of (F) MMP9/TIMP1 and (G) MMP2/TIMP2, respectively. ${ }^{~} \mathrm{P}<0.05$ vs. control and EP groups, "P<0.05 vs. ADR group. EP, ethyl pyruvate; ADR, Adriamycin; TGF $\beta-1$, transforming growth factor- $\beta 1$; Colla1, collagen type $1 \alpha 1$; Colla3, collagen type $1 \alpha 3$; MMP2, matrix metalloproteinase-2; MMP9, matrix metalloproteinase-9; TIMP1, tissue inhibitor of metalloproteinase-1; TIMP2, inhibitor of metalloproteinase-2. 
In conclusion, this study showed that EP could alleviate ADR-induced myocardial damage by preserving the diastolic relaxation and systolic contractile force of the heart. In addition, it blocked the source of ROS and thus resisted oxidative injury in myocardial tissue. Furthermore, EP prevented the apoptosis and fibrosis of myocardial tissues in ADR-treated rats. These findings indicate that EP is a potential novel therapeutic agent for ADR-induced cardiomyopathy.

\section{Acknowledgements}

This study was supported by grants to Dr J Wan from the National Natural Science Foundation of China (grant nos. 81170208 and 30871050), and the Natural Science Foundation of Hubei province, China (grant no. 302-131725).

\section{References}

1. Felker GM, Thompson RE, Hare JM, Hruban RH, Clemetson DE, Howard DL, Baughman KL and Kasper EK: Underlying causes and long-term survival in patients with initially unexplained cardiomyopathy. N Engl J Med 342: 1077-1084, 2000.

2. Zhang S, Liu X, Bawa-Khalfe T, Lu LS, Lyu YL, Liu LF and Yeh ET: Identification of the molecular basis of doxorubicin-induced cardiotoxicity. Nat Med 18: 1639-1642, 2012.

3. Jones LW, Haykowsky MJ, Swartz JJ, Douglas PS and Mackey JR: Early breast cancer therapy and cardiovascular injury. J Am Coll Cardiol 50: 1435-1441, 2007.

4. Arya DS, Bansal P, Ojha SK, Nandave M, Mohanty I and Gupta SK: Pyruvate provides cardioprotection in the experimental model of myocardial ischemic reperfusion injury. Life Sci 79: 38-44, 2006.

5. Sims CA, Wattanasirichaigoon S, Menconi MJ, Ajami AM and Fink MP: Ringer's ethyl pyruvate solution ameliorates ischemia/reperfusion-induced intestinal mucosal injury in rats. Crit Care Med 29: 1513-1518, 2001.

6. Sileri P, Schena S, Morini S, Rastellini C, Pham S, Benedetti E and Cicalese L: Pyruvate inhibits hepatic ischemia-reperfusion injury in rats. Transplantation 72: 27-30, 2001.

7. Yi JS, Kim TY, Kyu Kim D and Koh JY: Systemic pyruvate administration markedly reduces infarcts and motor deficits in rat models of transient and permanent focal cerebral ischemia. Neurobiol Dis 26: 94-104, 2007.

8. Marcengill MB, Puri S, Puri SK, Mohanram A, Leonova E, Raymond RM and Watts JA: Antioxidant effects of pyruvate in isolated rat hearts. J Mol Cell Cardiol 27: 2059-2067, 1995.

9. Kao KK and Fink MP: The biochemical basis for the anti-inflammatory and cytoprotective actions of ethyl pyruvate and related compounds. Biochem Pharmacol 80: 151-159, 2010.

10. Guo JL, Zhang J, Luo XY, Luo WM, Lin CY, Zhang KL and Ji YM: Effects of ethyl pyruvate on cardiac function recovery and apoptosis reduction after global cold ischemia and reperfusion. Exp Ther Med 7: 1197-1202, 2014.

11. Kao KK, Fink MP: The biochemical basis for the anti-inflammatory and cytoprotective actions of ethyl pyruvate and related compounds. Biochem Pharmacol 80: 151-159, 2010.

12. Clark JD, Gebhart GF, Gonder JC, Keeling ME and Kohn DF: The 1996 Guide for the Care and Use of Laboratory Animals. ILAR J 38: 41-48, 1997.

13. Jiang DS, Li L, Huang L, Gong J, Xia H, Liu X, Wan N, Wei X, Zhu $\mathrm{X}$, Chen Y, et al: Interferon regulatory factor 1 is required for cardiac remodeling in response to pressure overload. Hypertension 64: 77-86, 2014.

14. Desagher S, Glowinski J and Premont J: Astrocytes protect neurons from hydrogen peroxide toxicity. J Neurosci 16 2553-2562, 1996.
15. Shadab M, Agrawal DK, Aslam M, Islam N and Ahmad Z: Occupational health hazards among sewage workers: Oxidative stress and deranged lung functions. J Clin Diagn Res 8: BC11-BC12, 2014

16. Leontyev S, Schlegel F, Spath C, Schmiedel R, Nichtitz M, Boldt A, Rübsamen R, Salameh A, Kostelka M, Mohr FW and Dhein S: Transplantation of engineered heart tissue as a biological cardiac assist device for treatment of dilated cardiomyopathy. Eur J Heart Fail 15: 23-35, 2013.

17. Li K, Sung RY, Huang WZ, Yang M, Pong NH, Lee SM, Chan WY, Zhao H, To MY, Fok TF, et al: Thrombopoietin protects against in vitro and in vivo cardiotoxicity induced by doxorubicin. Circulation 113: 2211-2220, 2006.

18. Lau DH, Psaltis PJ, Carbone A, Kelly DJ, Mackenzie L, Worthington M, Metcalf RG, Kuklik P, Nelson AJ,Zhang Y, et al: Atrial protective effects of $n-3$ polyunsaturated fatty acids: A long-term study in ovine chronic heart failure. Heart Rhythm 8: 575-582, 2011.

19. Andreadou I, Mikros E, Ioannidis K, Sigala F, Naka K, Kostidis S, Farmakis D, Tenta R, Kavantzas N, Bibli SI, et al: Oleuropein prevents doxorubicin-induced cardiomyopathy interfering with signaling molecules and cardiomyocyte metabolism. J Mol Cell Cardiol 69: 4-16, 2014.

20. Ohkura K, Lee JD, Shimizu H, Nakano A, Uzui H, Horikoshi M, Fujibayashi Y, Yonekura Y and Ueda T: Mitochondrials complex I activity is reduced in latent adriamycin-induced cardiomyopathy of rat. Mol Cell Biochem 248: 203-208, 2003.

21. Brandes RP, Weissmann N and Schröder K: Nox family NADPH oxidases: Molecular mechanisms of activation. Free Radic Biol Med 76: 208-226, 2014.

22. West AP, Brodsky IE, Rahner C, Woo DK, Erdjument-Bromage H, Tempst P, Walsh MC, Choi Y, Shadel GS and Ghosh S: TLR signalling augments macrophage bactericidal activity through mitochondrial ROS. Nature 472: 476-480, 2011.

23. Maejima Y, Kuroda J, Matsushima S, Ago T and Sadoshima J: Regulation of myocardial growth and death by NADPH oxidase. J Mol Cell Cardiol 50: 408-416, 2011.

24. Zhao Y, McLaughlin D, Robinson E, Harvey AP, Hookham MB, Shah AM, McDermott BJ and Grieve DJ: Nox2 NADPH oxidase promotes pathologic cardiac remodeling associated with doxorubicin chemotherapy. Cancer Res 70: 9287-9297, 2010.

25. Ortiz C, Caja L, Sancho P, Bertran E and Fabregat I: Inhibition of the EGF receptor blocks autocrine growth and increases the cytotoxic effects of doxorubicin in rat hepatoma cells: Role of reactive oxygen species production and glutathione depletion. Biochem Pharmacol 75: 1935-1945, 2008.

26. Gao S, Li H, Feng XJ, Li M, Liu ZP, Cai Y, Lu J, Huang XY, Wang JJ, Li Q, et al: $\alpha$-Enolase plays a catalytically independent role in doxorubicin-induced cardiomyocyte apoptosis and mitochondrial dysfunction. J Mol Cell Cardiol 79: 92-103, 2015.

27. Janeesh PA and Abraham A: Robinin modulates doxorubicin-induced cardiac apoptosis by TGF- $\beta 1$ signaling pathway in Sprague Dawley rats. Biomed Pharmacother 68: 989-998, 2014.

28. Wight TN and Potter-Perigo S: The extracellular matrix: An active or passive player in fibrosis? Am J Physiol Gastrointest Liver Physiol 301: G950-G955, 2011.

29. Sethi A, Jain A, Zode GS, Wordinger RJ and Clark AF: Role of TGFbeta/Smad signaling in gremlin induction of human trabecular meshwork extracellular matrix proteins. Invest Ophthalmol Vis Sci 52: 5251-5259, 2011.

30. Xu J, Wang P, Wang T, Wang M, Chen S, Yu P and Yu D: Effects of reduced $\beta 2$-glycoprotein I on the expression of aortic matrix metalloproteinases and tissue inhibitor matrix metalloproteinases in diabetic mice. BMC Cardiovasc Disord 14: 114, 2014

31. Arafa MH, Mohammad NS, Atteia HH and Abd-Elaziz HR: Protective effect of resveratrol against doxorubicin-induced cardiac toxicity and fibrosis in male experimental rats. J Physiol Biochem 70: 701-711, 2014. 